\title{
Sistema Espacial de Suporte à Decisão para Gestão do Combate ao Dengue usando Lógica Fuzzy
}

\author{
J.C.S. MELO ${ }^{1}$ e R.M. MORAES ${ }^{2}$ \\ Recebido em 31 de Março de 2017 / Aceito em 10 de Abril de 2018
}

\begin{abstract}
RESUMO. Um grande problema de saúde pública brasileiro é a dengue, cujo vírus é transmitido por um vetor (o mosquito Aedes aegypt). Apesar dos esforços em controlar essa doença, o número de casos registrados tem aumentado com o passar dos anos e isso leva a um problema epidemiológico de identificar áreas prioritárias para combatê-la e dar suporte à definição de políticas públicas de saúde específicas. Este trabalho visa o desenvolvimento de um sistema de suporte à decisão baseado em informação espacial, que permita levar em consideração diferentes informações agregadas por um sistema baseado em regras fuzzy para detectar áreas de prioridade de combate a esse agravo. O sistema foi aplicado ao estudo do dengue no estado da Paraíba entre os anos de 2009 a 2013.
\end{abstract}

Palavras-chave: Lógica Fuzzy, Sistema de Suporte a Decisão Espacial, Dengue.

\section{INTRODUÇÃO}

Um dos maiores problemas de saúde pública da atualidade é a disseminação da dengue. Ela ocorre por meio de diversos fatores, entre eles está o aumento na intensidade e velocidade do tráfego de pessoas entre países e/ou continentes. A dengue é rapidamente difundida pelo vetor (o mosquito Aedes aegypt), que se alimenta do sangue de pessoas infectadas e retransmite a doença de pessoa a pessoa [8]. O vírus pode ser detectado no sangue até dois dias antes do aparecimento dos primeiros sintomas e até oito dias após o seu início. É dentro desse intervalo de tempo que o Aedes Aegypti pode contrair o vírus do indivíduo infectado e transmiti-lo para outro (os) [22].

O dengue é categorizado em: DENV-1, DENV-2, DENV-3 e DENV-4 [2]. Na época da escravidão no Brasil, o Aede Aegypti chegou ao país, vindo nos navios negreiros. Ele foi erradicado do país na década de 50, em uma agressiva campanha de combate a febre amarela, da qual ele também é vetor. No fim da década de 70, novamente foi detectada sua presença nas principais metrópoles

\footnotetext{
*Autor correspondente: Ronei Marcos de Moraes - E-mail: ronei@ de.ufpb.br

${ }^{1}$ Laboratório de Estatística Aplicada ao Processamento de Imagens e Geoprocessamento, Departamento de Estatística, CCEN, Universidade Federal da Paraíba, Cidade Universitária s/n, 58.051-900, João Pessoa, PB, Brasil. E-mail: zehk07@gmail.com

2 Departamento de Estatística, CCEN, Universidade Federal da Paraíba, Cidade Universitária s/n, 58.051-900, João Pessoa, PB, Brasil. E-mail: ronei@ de.ufpb.br
} 
brasileiras. Já a epidemia da doença se alastrou a partir do ano de 1986, com a recirculação do DENV-1, no Estado do Rio de Janeiro, propagando-se, logo em seguida, para a região Nordeste. Em 1990, no mesmo Estado, houve um aumento na resistência da doença contra os anticorpos utilizados até o momento, sendo detectado um novo sorotipo, o DENV-2, acarretando os primeiros diagnósticos de FHD (Febre Hemorrágica da Dengue) [23]. Já em 2001, foi incorporado nos Estados de Roraima e Rio de Janeiro o DENV-3, que foi responsável pelo grande surto de dengue no ano seguinte, com elevado número de óbitos; atualmente circula em 25 das 27 unidades federativas. O quarto sorotipo da doença ressurgiu no ano de 2008 em Manaus, no Amazonas [14].

Atualmente esse mosquito é encontrado em mais de 4000 municípios brasileiros. A quantidade de casos registrados vêm aumentando com o passar dos anos e acarreta um problema epidemiológico de identificar áreas prioritárias para combatê-los e também prover suporte à definição de políticas públicas de saúde específicas [14]. Essa questão remete a necessidade em detectar onde os casos estão localizados espacialmente. Sistemas de Suporte a Decisão Espacial (em inglês, SDSS) [20] podem ser delineados para a identificação e classificação de regiões de acordo com níveis de prioridade de tais regiões geográficas, baseados em detecção de aglomerados espaciais [5] e usando regras fuzzy [17]. Este SDSS, por sua vez, fornece uma potencial plataforma para processar informações espaciais [11]. O suporte computacional para aplicações espaciais utilizando um SDSS é fornecido por sistemas, que são baseados em um Sistema de Informação Geográfica (SIG), que é um sistema computacional que facilita a exibição e armazenamento de dados geograficamente ou espacialmente relacionados, permitindo a integração desses dados com dados não espaciais [10]. Com isso, o SDSS permite subsidiar a decisão sobre a definição de municípios prioritários, visando à construção de políticas ou programas específicos, pois uma vez identificada à região o gestor de saúde pública pode tomar medidas de prevenção necessárias [17].

Dessa forma, o objetivo deste estudo é a utilização de um SDSS que possa considerar diferentes fatores relacionados a uma situação de incerteza através da aplicação de um sistema baseado em regras fuzzy, detectando as áreas de prioridade para os casos do dengue dos municípios do Estado da Paraíba entre os anos de 2009 a 2013. Seu uso pode otimizar recursos financeiros e de pessoal, direcionando-os para onde eles são mais necessários [13]. Com a finalidade de favorecer a tomada de decisão sobre políticas públicas de prevenção, foi utilizada uma adaptação da arquitetura de SDSS [18], que faz uso dos fatores associados a esta situação.

\section{METODOLOGIA}

Diversas arquiteturas de SDSS podem ser encontradas na literatura científica [10] [21] [25] [7]. Entretanto, são poucas as direcionadas especificamente à epidemiologia [11]. Recentemente, uma arquitetura modular foi proposta para suporte à decisão sobre problemas epidemiológicos [18]. Ela foi utilizada neste trabalho e sua estrutura pode ser visualizada em detalhes na Figura 1. Ela permite utilizar módulos de análises estatísticas (não espaciais), espaciais e espaço-temporais para análises preliminares sobre vários tipos de informações e agregá-las usando um sistema ba- 
seado em regras fuzzy, que permite a geração de mapas de decisão. Nesta arquitetura não se faz necessária a utilização de todos os módulos, ou seja, o mapa de decisão pode ser gerado com apenas uma parte deles. Os mapas gerados com base nessa arquitetura têm por objetivo orientar os gestores de saúde em tomada de decisões, como a intervenção ou não sobre determinadas doenças, e definir as áreas onde ela está ocorrendo, para que assim sejam tomadas as providências necessárias.

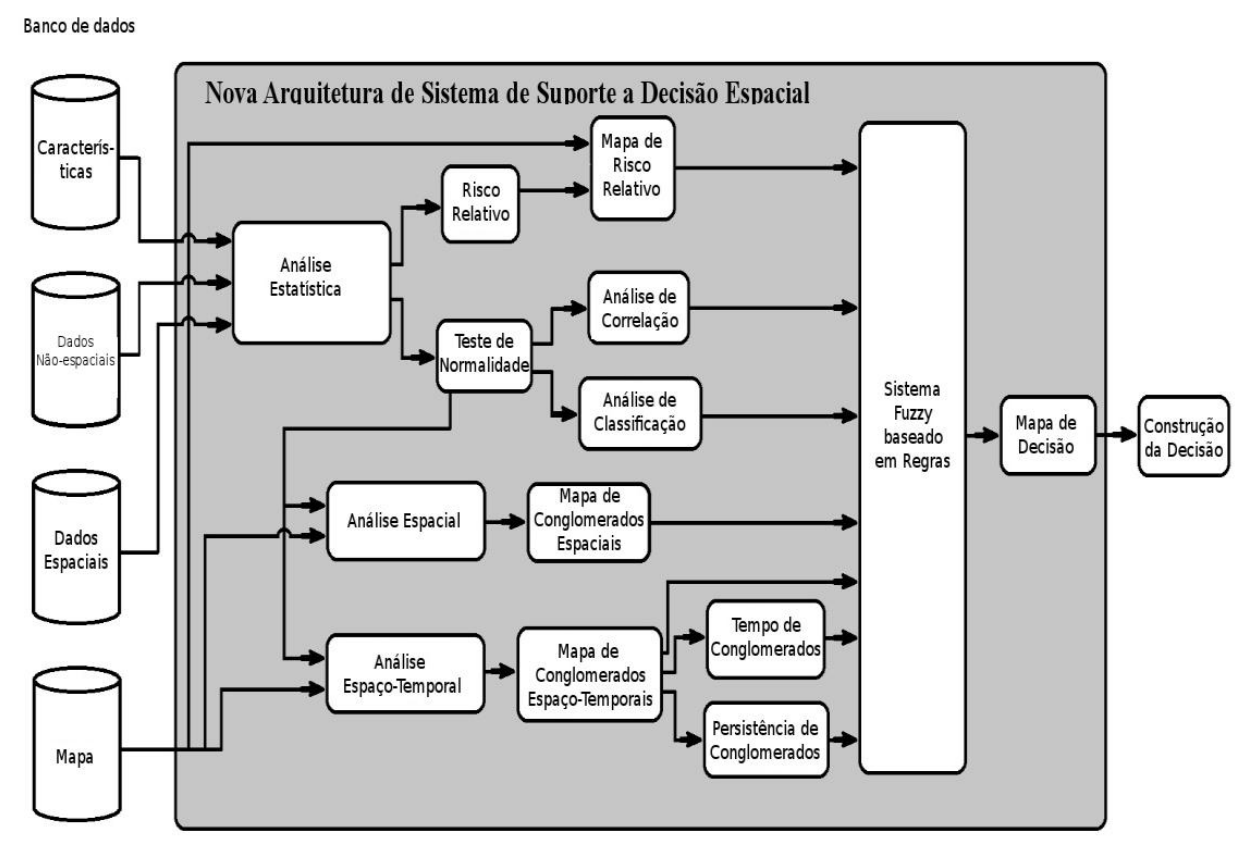

Figura 1: Arquitetura de SDSS [18].

Essa arquitetura é flexível e permite que apenas alguns módulos sejam utilizados na solução de problemas, de acordo com a sua especificidade e necessidade. Para este caso, foram utilizados os módulos de Risco Relativo (RR), o de Análise Espacial e o de Sistema Baseado em Regras Fuzzy, sendo o motivo pela escolha à tomada de decisão sobre a epidemiologia anual do dengue. Os dois primeiros servirão de entrada para o módulo de Sistema Baseado em Regras Fuzzy, gerando ao final, de acordo com a arquitetura, mapas de decisões.

A fim de melhorar a compreensão sobre cada módulo utilizado da arquitetura, tem-se que uma região geográfica $G$ é uma área geográfica de interesse, na qual o estudo epidemiológico é realizado. Do ponto de vista da área de geoprocessamento, se a região geográfica é um Estado, ela pode ser dividida em sub-regiões que podem ser os municípios, os bairros, ou mesmo as casas que os compõem, por exemplo. Essas entidades são objetos distintos e localizáveis dentro da região geográfica e são chamados de geo-objetos $g_{i}^{\prime} s$ [13], onde $g_{i}^{\prime} s \in G$. 
No módulo de RR é calculado o risco, o qual permite comparar informações de diferentes $g_{i}^{\prime} s$, pois padroniza os dados retirando, com isso, o efeito das diferentes populações. Este indicador, de acordo com Lucena [14], representa a intensidade da ocorrência de um fenômeno com relação a todas as regiões de estudo, estas regiões, por sua vez, seriam os municípios da Paraíba. A equação do RR é denotada por:

$$
R R_{i}=\frac{\frac{x_{i}}{n_{i}}}{\frac{\Sigma x_{i}}{\Sigma n_{i}}}
$$

onde $x_{i}$ é o número de ocorrência do fenômeno em um município do Estado e $n_{i}$ é a população desse município.

No módulo de análise espacial, são diversos os instrumentos que podem ser utilizados para determinar a localização espacial dos casos do dengue, como os métodos espaciais Scan [12], Scan Flexível [26], Besag e Newell [3] e Getis e Ord [1]. A análise espacial utilizada no modelo foi a do método Scan, proposto por Kulldorff e Nagarwalla.

O método Scan, por sua vez, atribui círculos de raio arbitrário para cada centroide (centro de um geo-objeto) e faz uma busca por toda região para encontrar áreas cuja ocorrência de um fenômeno seja significativamente diferente da sua vizinhança. Logo após, calcula o número de ocorrências dentro desse círculo [4], se o valor observado de ocorrências for maior que o esperado, a área demarcada pelo círculo é chamada de conglomerado. Caso contrário, o raio do círculo é aumentado até envolver outro centroide ou até que o parâmetro da estimativa da população sob risco seja atingida. O processo se repete até todos os centroides serem testados e a hipótese nula é de que não há conglomerados na região de estudo [12]. Como não é possível conhecer o verdadeiro valor da população sob risco, toma-se como referência o mapa de RR [16] [9], para sua obtenção.

O último módulo fará uso da lógica fuzzy, que tem por objetivo a modelagem computacional da imprecisão do raciocínio humano [28]. A sua fundamentação matemática encontra-se na teoria dos conjuntos fuzzy, onde se afirma que dado um determinado elemento que pertence a um domínio, é verificado o grau de pertinência do elemento em relação ao conjunto. O grau de pertinência serve como referência para verificar o quanto "é possível" esse elemento pertencer ao conjunto. $\mathrm{O}$ grau é calculado através de uma determinada função de pertinência que retorna um valor real que varia entre 0 a 1 , sendo que 0 indica que não pertence ao conjunto, e 1 indica que pertence totalmente [27].

A diferença entre a lógica fuzzy e a lógica booleana é que a booleana tem como princípio usar valores binários para modelagem de problemas do tipo "Se o copo está cheio, então, posso matar minha sede"; ou de outra forma: "Se o copo está vazio, então não posso matar minha sede". A lógica fuzzy é capaz de lidar com situações imprecisas para solucionar problemas do tipo "Se o copo está quase vazio então posso matar parte da minha sede". Por conseguinte, regras fuzzy são utilizadas para se atuar sobre os conjuntos fuzzy, fazendo uso de um raciocínio lógico impreciso, com o intuito de obter consequentes.

Sistemas baseados em regras fuzzy utilizam conectivos do tipo AND (E) e OR (OU), que são equivalentes às operações de intersecção e união de conjuntos fuzzy respectivamente. Estes po- 
dem aparecer tanto na premissa (condição) quanto na conclusão de uma dada regra. A condição representa o argumento de uma regra e a conclusão remete ao resultado final, que no caso do estudo representam os graus de prioridade. São dois os sistemas de inferência mais conhecidos fuzzy (em inglês, FIS): o de Mamdani, que é baseado numa estrutura simples de operações min-max, envolvendo regras de inferência do tipo: Se $x$ é $A$ E $y$ é $B$ Então $z$ é $C$ onde $A, B$ e $C$ são conjuntos fuzzy [15]; e o de Takagi-Sugeno, que consiste em um sistema em que a saída não remete a um conjunto fuzzy e sim a uma função fuzzy [24]. Neste trabalho foi usado o FIS de Mamdani, com processo de "defuzzificação", que permite representar um conjunto fuzzy por meio de um número real, no qual os dados fuzzy são convertidos em valores numéricos precisos, utilizando, dentre os métodos existentes, o método do centroide a fim de se obter a saída do sistema [20].

Vale ressaltar que a entrada do Sistema Baseado em Regras Fuzzy (SBRF) é dada sobre a medida de Risco Relativo do dengue para cada município em cada ano estudado, bem como a saída do modelo estatístico de aglomeração espacial. Os dados brutos do dengue em si não foram utilizados como entrada para o SBRF. Desse modo, as regras versam sobre a análise automatizada conjunta da medidade de Risco Relativo e do modelo estatístico, cujo conhecimento foi oriundo de um especialista.

A de base de dados obtida a partir da parceria com a Secretaria de Saúde do Estado da Paraíba, refere-se aos casos registrados do dengue em cada município do Estado entre os anos de 2009 e 2013. A partir dela pode-se obter as informações necessárias para a aplicação da arquitetura. Para o caso estudado os dados de entrada para a arquitetura são: número de casos notificados de dengue no período, a população sob risco no mesmo período, as coordenadas geográficas dos municípios em questão e o mapa cartográfico digital da região geográfica em análise.

Sendo um processo empírico, verifica-se manualmente a relação da distribuição dos dados com cada decisão possível. Apesar de existirem diversas formas, é comum se basear no histograma dos dados da condição e da conclusão. Dados amostrais são utilizados para extrair histogramas das diversas variáveis, respeitando o domínio original das variáveis e o seu formato final é semelhante ao fornecido pelo histograma. Ao final, a informação contida nas variáveis originais são similares às funções de pertinência dos conjuntos fuzzy [20]. Portanto, a partir dessas relações, constroem-se regras envolvendo conjuntos fuzzy no antecedente e no consequente.

Desta maneira, são construídas regras a fim de se checar se, na prática, o resultado gerado é relativo ao que de fato é esperado para aquela determinada situação. As regras que denotam um mesmo contexto podem ser unidas de modo a melhorar o conhecimento, produzindo regras complexas. A vantagem dessa abordagem é a criação de conjuntos de regras que produzem decisões de modo coerente e que são plenamente justificadas pelas bases de dados de onde o conhecimento foi extraído [13]. O processo só chega ao fim quando não há a possibilidade de criar regras complexas.

Os resultados obtidos são utilizados para se construir uma espacialização, ou seja, utilizados para se gerar mapas coropléticos (coloridos) para melhor visualizar toda a região geográfica $G$ sobre o 
qual o fenômeno ocorre. Essas informações podem ser oriundas de subdivisões de $G$, e a partir de análises estatísticas espaciais, podemos analisar individualmente essas subdivisões $\{[13]$. Com a tomada de decisão se pode fazer uso dessas informações para se chegar a uma análise espacial da região de estudo e reuni-las em mapas coropléticos, dando origem ao SDSS [17] [25] .

\section{RESULTADOS}

O espaço de decisões para o SDSS usando regras fuzzy é dado pelo conjunto: \{ "Não prioritário", "Possivelmente não prioritário", "Possivelmente prioritário" e "Prioritário"\}, o qual representa todos os níveis de prioridade em que um município pode ser classificado. Com isso, é possível que o modelo informe a condição dos municípios que devem ser prioritários, orientando o gestor de saúde na sua tomada de decisão. Um exemplo das funções de pertinência para os módulos abordados pode ser observado na Figura 2.

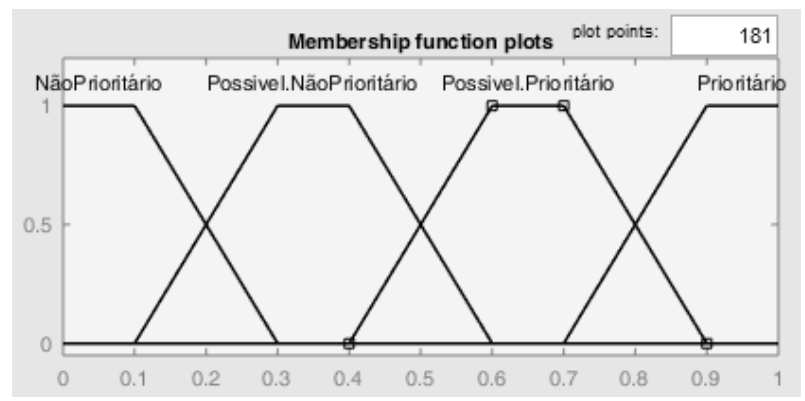

Figura 2: Função de pertinência para a decisão final.

Para a construção da função de pertinência do RR foi utilizado o formato trapezoidal, como visto na Figura 3, enquanto para a função de pertinência do método Scan, o formato triangular foi utilizado, Figura 4. A partir das funções e da base de dados foram construídas as regras, sendo um total de 10. Como exemplo das regras utilizadas tem-se:

Regra 1. IF (Risco Relativo alto) AND (Não é um conglomerado espacial) THEN (Município é Possivelmente prioritário).

Regra 4. IF (Risco Relativo baixo) AND (É um conglomerado espacial) THEN (Município é não prioritário).

Regra 5. IF (Risco Relativo muito alto) AND (É um conglomerado espacial) THEN (Município é prioritário).

A exemplo da quinta regra, ela pode ser interpretada da seguinte forma: "SE o Risco Relativo do município for muito alto E ele for detectado como um conglomerado espacial, ENTÃO este município é prioritário". As demais seguem este formato de interpretação, obtendo ao final todos os graus de pertinência que serão utilizados no estudo. 


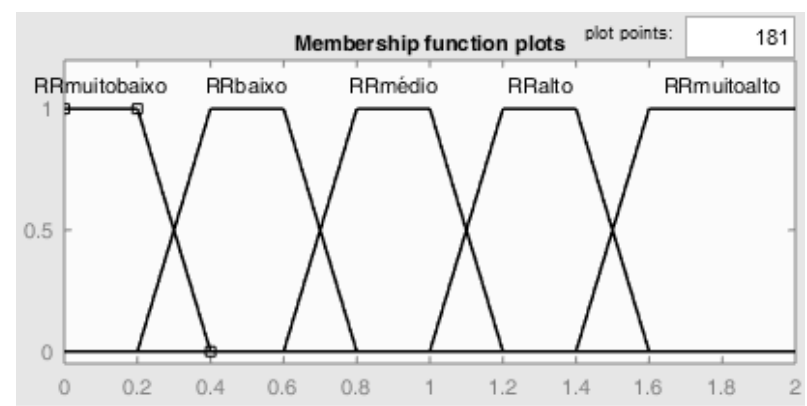

Figura 3: Função de pertinência para o Risco Relativo.

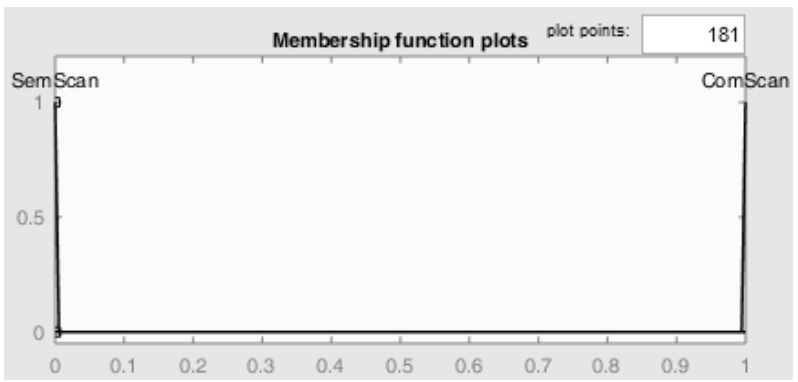

Figura 4: Função de pertinência para o método Scan.

Com isso, fazendo uso das regras, tem-se a decisão de prioridade dos municípios abordados para os anos de 2009 a 2013. A saída da decisão final pode ser observada na Figura 5.

Foram realizadas 1115 aplicações para contemplar os 223 municípios da Paraíba nos cinco anos abordados, para no fim serem gerados os cinco mapas de decisão, sendo um para cada ano. Tais mapas que foram gerados a partir de um modelo de decisão que leva em conta o risco relativo, a análise espacial e o sistema fuzzy baseado em regras, no qual indica a prioridade para cada um dos municípios paraibanos. Como não se conhece o verdadeiro resultado do processo, toma-se como referência o mapa de RR [16] [9].

Observando o mapa de RR na Figura 6, constata-se que grande parte dos municípios apresentaram baixo valor de risco, sendo mais concentrado na parte leste do mapa, representando a parte litorânea do estado. Não obstante, nota-se uma concentração de municípios de alto risco nas regiões sudeste, noroeste e nordeste do estado.

No mapa de decisão de 2009 (Figura 7), verifica-se um espalhamento dos municípios considerados prioritários na parte central e oeste do estado, bem como uma pequena concentração de municípios prioritários na parte nordeste do mesmo. A maioria dos municípios foi considerada como "não prioritário" e "com tendência a não prioritário". Em contrapartida quinze municípios foram sinalizados como possivelmente prioritários. Para este ano, foram detectados vinte e sete 


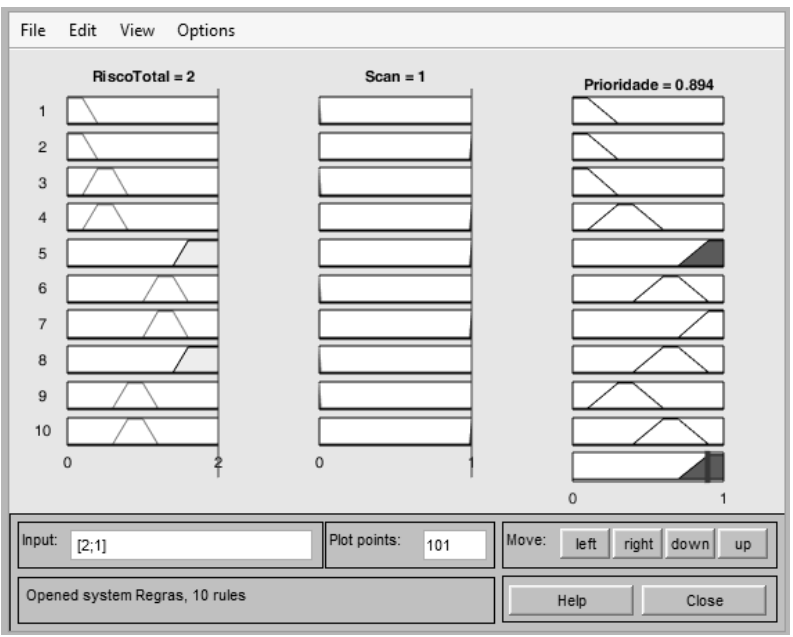

Figura 5: Aplicação das regras para o total do ano de 2010 no município de Cabedelo - PB.

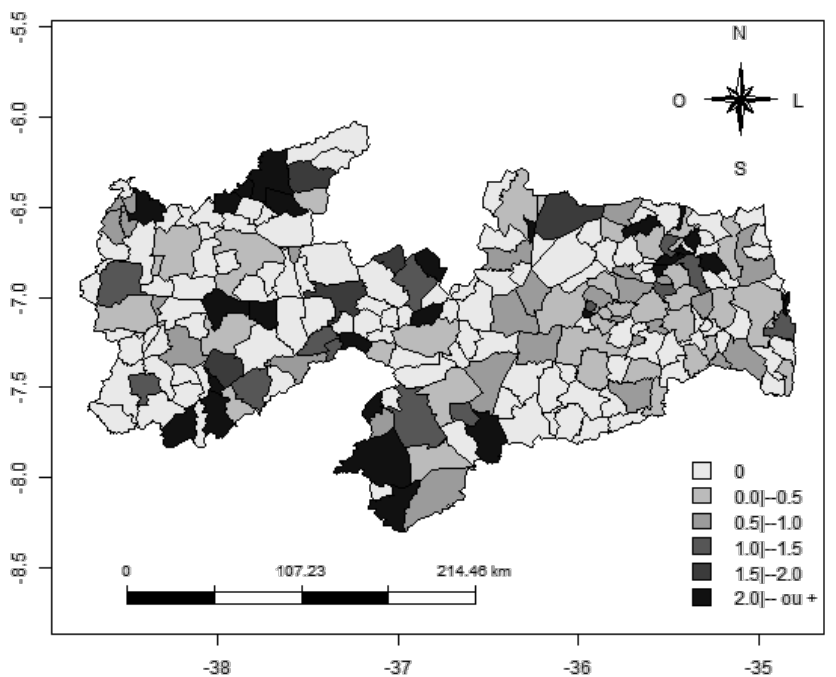

Figura 6: Mapa de risco para o ano de 2009.

municípios como "prioritários". Ao comparar os mapas de RR e de decisão, se pode averiguar a conformidade do mapa de decisão em detectar a prioridade dos municípios.

Diferentemente da ideia de aglomeração não espacial, a existência de hetorogeneidade na aglomeração é possível, pois está relacionada com a significância estatística, ou não, do risco 


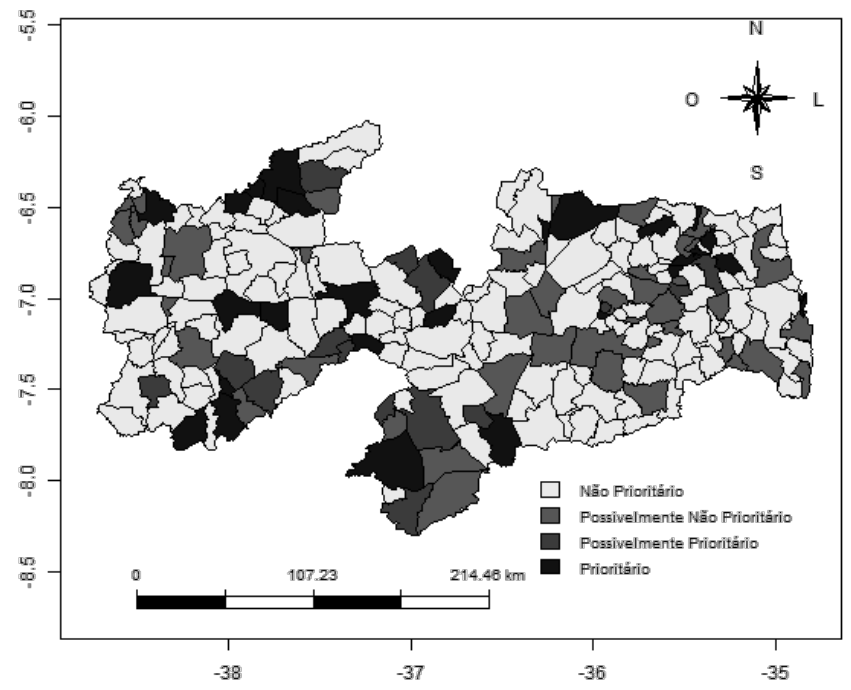

Figura 7: Mapa de decisão para o ano de 2009.

observado naquela localidade. Já do ponto de vista epidemiológico, áreas de risco baixo ou nulo próximas a áreas de risco elevado denotam áreas de proteção. Em Epidemiologia, as "áreas de proteção" se referem as áreas onde um determinado agravo ocorre em graus de severidade muito menores do que em sua vizinhança, ou seja, implica regiões que por alguma causa natural ou não, registram valores diferentes do seu entorno. As áreas identificadas nos mapas apresentados seguem essa premissa. [19]

Observando o mapa de RR para o ano de 2010 (Figura 8), tem-se que ao leste existe um grande número de municípios com baixo valor de risco. Também pode-se observar uma concentração de municípios de alto risco na região a oeste do estado. Se compararmos os resultados de ambos os mapas, observa-se que o mapa de decisão foi preciso quanto à especificação dos níveis de prioridade para os municípios.

Para o ano de 2010 (Figura 9), nota-se que existe um grande número de municípios em que não foi detectada prioridade, segundo o mapa de decisão, tendo uma concentração de municípios prioritários na parte oeste do mapa. Apenas quatro municípios foram sinalizados como possivelmente prioritários, uma quantidade inferior comparada ao número de possivelmente não prioritários. Neste ano se teve uma concentração de municípios não prioritários na parte leste do mapa, podendo isto ser indicação de algum fator de proteção. Entretanto, cerca de doze municípios apresentaram prioridade neste ponto cardeal.

Nota-se que o risco para o ano de 2011 (Figura 10) foi mais elevado quando comparado ao ano anterior. Poucos são os municípios que apresentaram risco baixo ou nulo, representados na parte sul do mapa. Comparando ao mapa de decisão, tem-se que a grande maioria dos municípios de alto risco pôde ser bem representada utilizando tal método. 


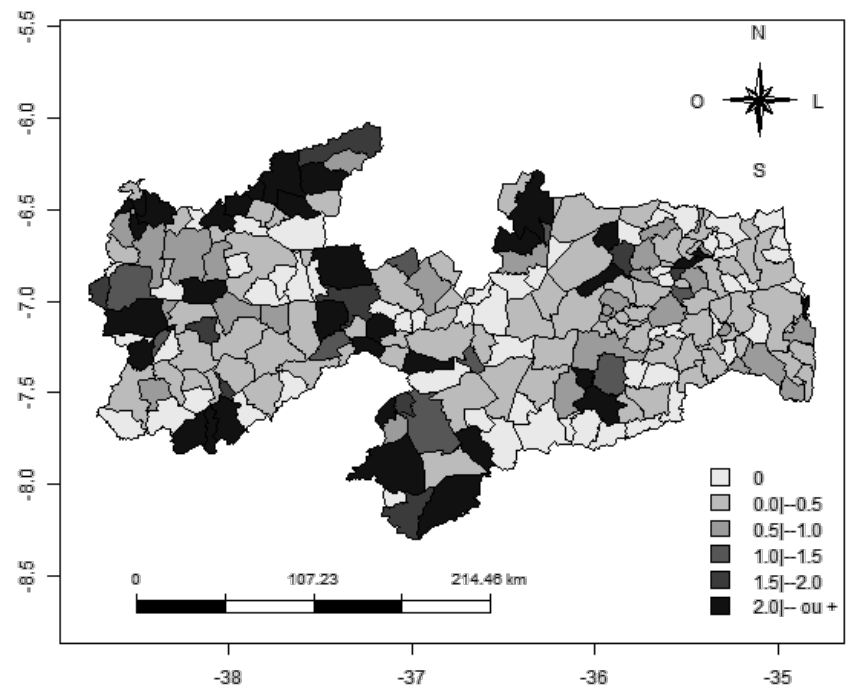

Figura 8: Mapa de risco para o ano de 2010.

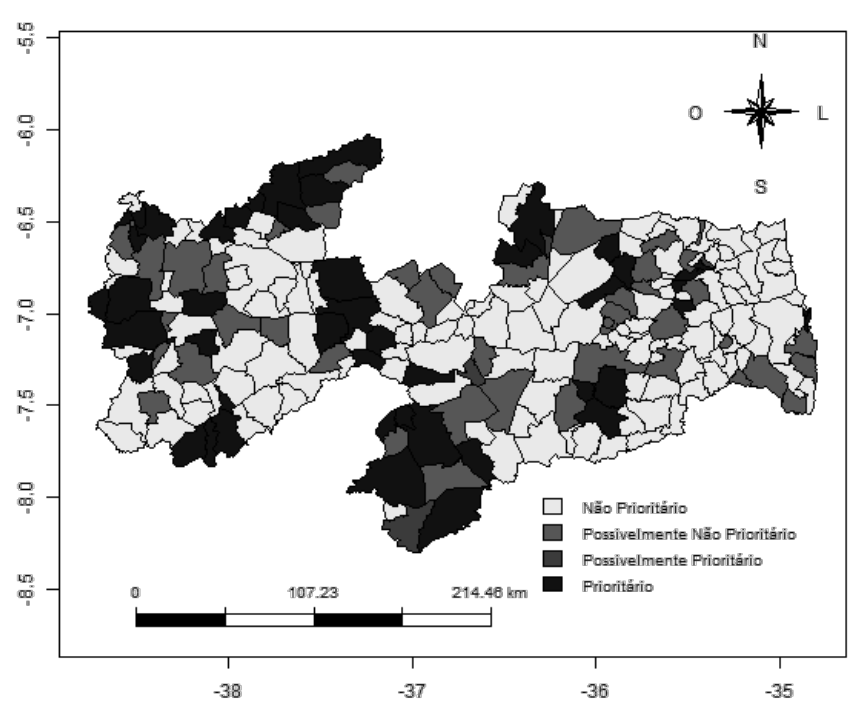

Figura 9: Mapa de decisão para o ano de 2010.

Analisando o mapa de decisão tem-se uma diminuição numérica nos municípios não prioritários, constatando-se que estes municípios estão mais dispersos no mapa apresentado na Figura 11. Neste ano a área norte do estado foi a que mais apresentou municípios prioritários, enquanto a área sudeste apresentou um aglomerado de municípios não prioritários. 


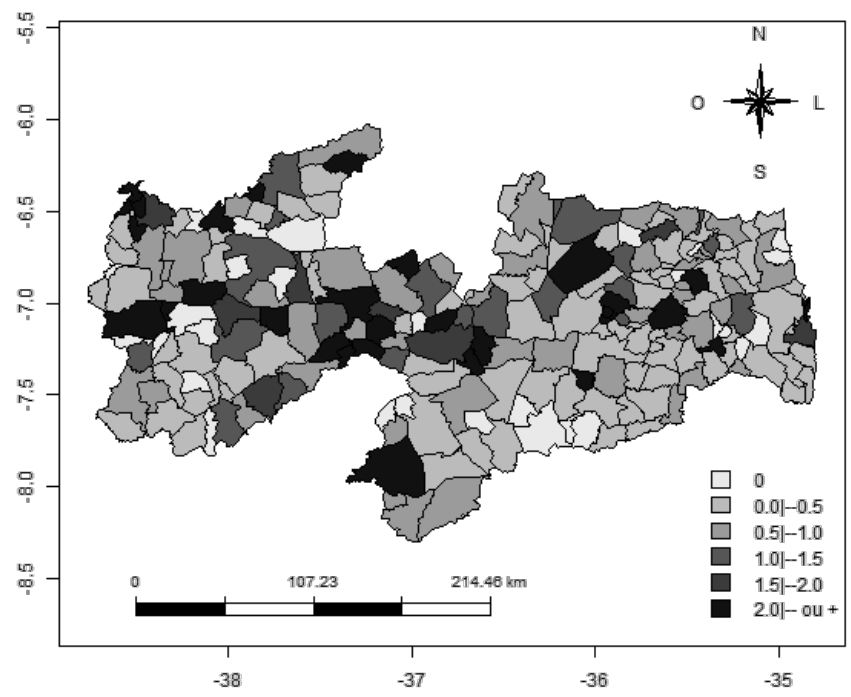

Figura 10: Mapa de risco para o ano de 2011.

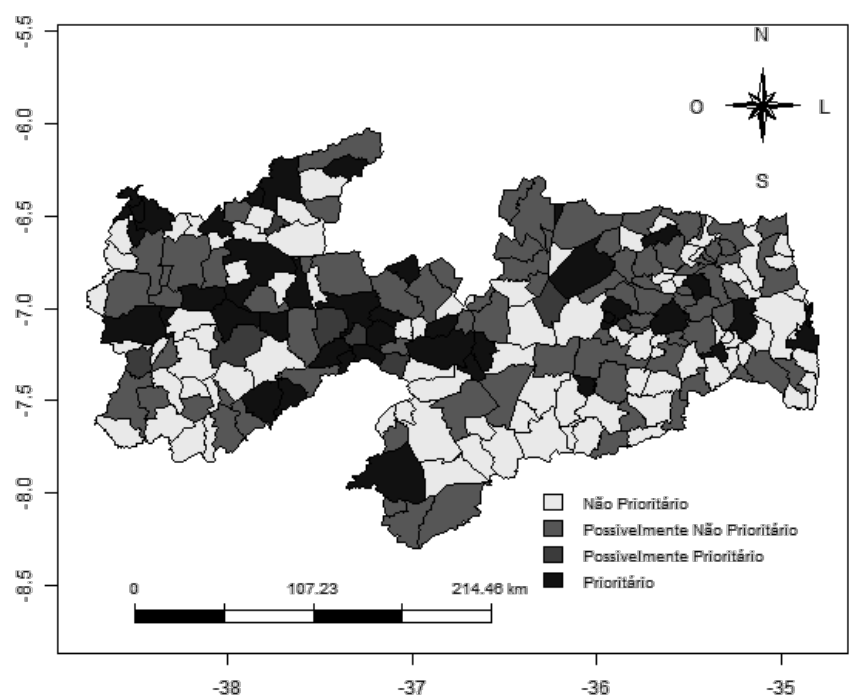

Figura 11: Mapa de decisão para o ano de 2011.

Analisando o mapa de RR para o ano de 2012 (Figura 12), mesmo com a diminuição dos municípios considerados de risco elevado, ainda existe uma maior quantidade de municípios de alto risco em comparação ao ano de 2010. O centro-norte do estado apresentou uma aglomeração de municípios de alto risco, contendo, em sua parte oeste, um maior número de municípios com risco elevado. 


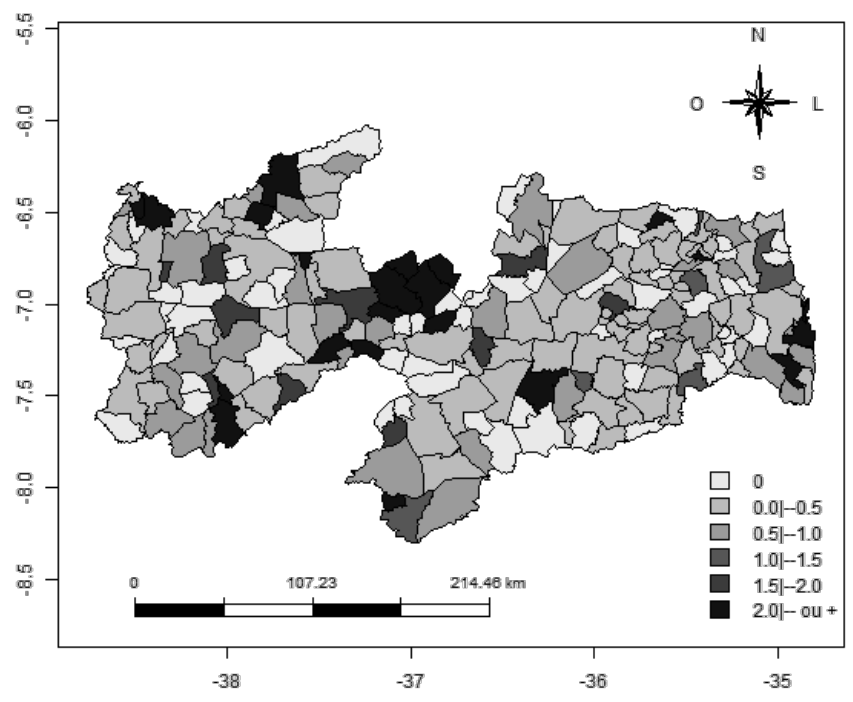

Figura 12: Mapa de risco para o ano de 2012.

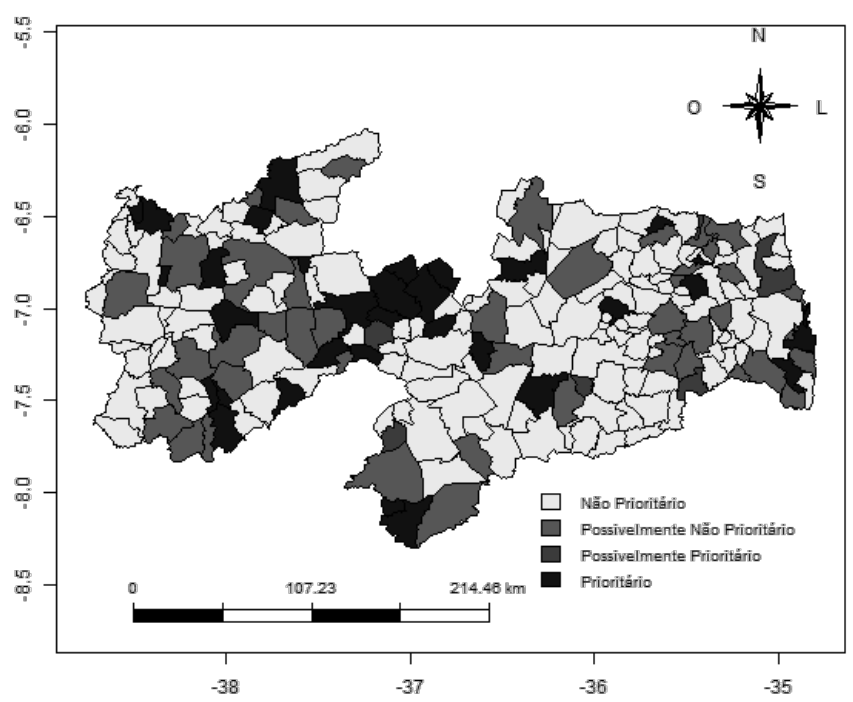

Figura 13: Mapa de decisão para o ano de 2012.

O ano de 2012 apresentou uma numérica diminuição nos municípios considerados não prioritários (Figura 13), constatando-se que estes municípios estão mais dispersos no mapa. Neste ano a parte norte do estado foi a que mais apresentou prioridade, enquanto a parte sudeste apresentou um aglomerado de municípios não prioritários. A dispersão quanto aos municípios pri- 
oritários é evidente, embora tenha uma concentração na região central do mapa. Novamente o mapa de decisão obteve exito em detectar tais municípios.

De acordo com o Ministério da Saúde, a diminuição dos números relativos à dengue é resultado de ações do governo, pois neste ano foram repassados $\mathrm{R} \$ 92,8$ milhões a 1.158 municípios para ações de prevenção e controle da doença, "No ano passado decidimos colocar $20 \%$ a mais de recurso nos municípios desde que fossem cumpridas as exigências na vigilância da saúde”, disse o Ministro da Saúde [6].

Observando o mapa de RR para o ano de 2013 (Figura 14) é visto que, em comparação aos anos anteriores, um número maior de municípios apresentou valor elevado de risco, sendo uma pequena minoria considerada de risco baixo ou nulo, representados, estes, nas partes sul, sudeste e leste do mapa.

No mapa de decisão (Figura 15) nota-se o aumento dos municípios prioritários, em sua maioria na região oeste do mapa adentrando na parte centro-leste. Por outro lado, a parte sudeste e leste apresentou uma concentração de municípios não prioritários, novamente podendo indicar uma área de proteção. Comparando-o ao mapa de RR, pode-se corroborar os resultados obtidos através do SDSS, uma vez que o risco relativo pôde ser bem representada utilizando tal método.

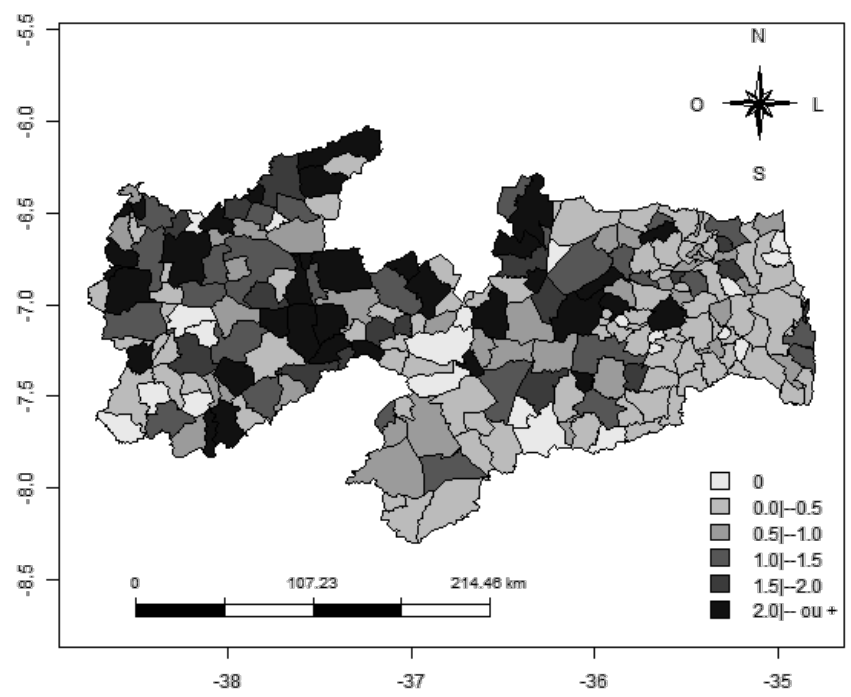

Figura 14: Mapa de risco para o ano de 2013.

\section{DISCUSSÃO}

O Sistema de Suporte a Decisão Espacial envolve a escolha da melhor decisão se baseando em um problema específico, que neste caso foram os casos de dengue na Paraíba. Levando em consideração tal aspecto epidemiológico, pode-se construir um modelo capaz de tratar dados 


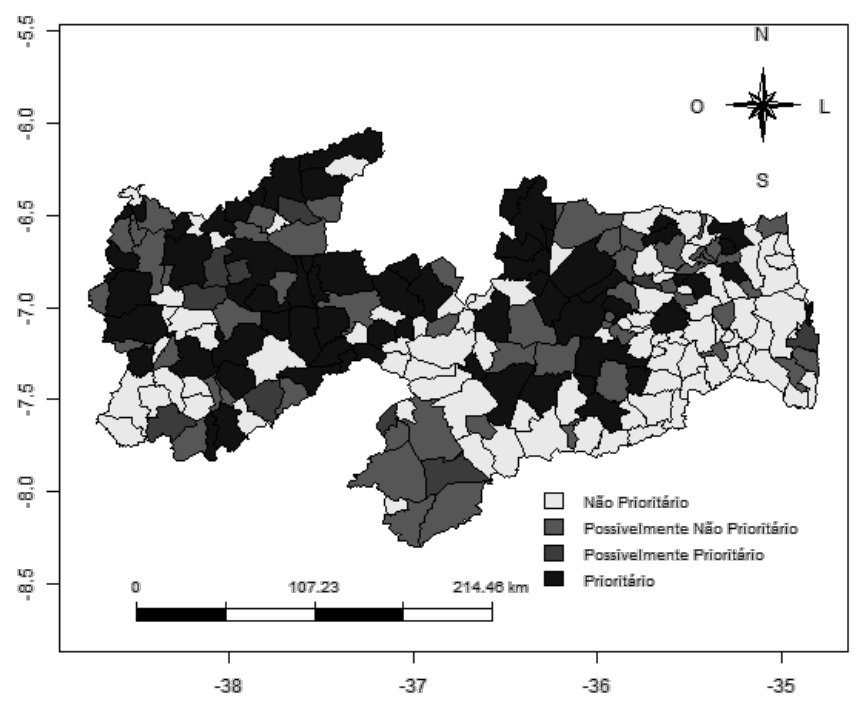

Figura 15: Mapa de decisão para o ano de 2013.

representados por elementos de área, fornecendo, com isso, mapas de decisão com diferentes níveis de prioridade.

Nessa perspectiva, problemas geográficos geralmente necessitam de tecnologias de suporte e o SDSS foi eficiente na tomada de decisão da prioridade dos municípios, utilizando-se, para isso, de regras fuzzy. O modelo também foi útil em reconhecer o padrão da distribuição geográfica da dengue, podendo, assim, auxiliar o gestor de saúde para se ter um maior controle da doença, reconhecendo as áreas de maior importância e estudando aquelas que apresentaram algum fator de proteção.

Do ponto de vista estrutural, o modelo faz uso de métodos espaciais somados a regras fuzzy, ou seja, ele consegue agregar formas de análise espacial utilizando regras e, assim, especificando melhor os resultados obtidos, uma vez que a decisão será tomada com base nos métodos de entrada do modelo. Levando em conta esta característica, os mapas gerados a partir de um SDSS são mais precisos e concisos. Um proveito do modelo utilizado é que não há a necessidade de trabalhar com todos os métodos de entrada, podendo escolher o tipo de entrada do mesmo.

\section{CONCLUSÃO}

Este trabalho fez uso da arquitetura de suporte à tomada de decisão baseado em regras fuzzy [18] para a modelagem a decisão sobre os níveis de prioridade para a intervenção dos municípios da Paraíba que notificaram casos do dengue nos anos de 2009 a 2013. O modelo possui a capacidade de considerar diferentes fatores que envolvem o problema em questão, fazendo uso destes para a tomada de decisão. Esse trabalho tem por objetivo melhor identificar os locais considerados prio- 
ritários pelo modelo, subsidiando de forma científica o processo de desenvolvimento de políticas públicas.

Existem na literatura diversos métodos espaciais capazes de lidar com tal problemática, mas este trabalho é o primeiro ao se basear nesta arquitetura, que faz uso de um sistema baseado em regras fuzzy, para o estudo de casos do dengue na Paraíba. Portanto, levando em consideração os resultados obtidos com a aplicação desta metodologia, foi constatado que ela tem o potencial de suportar decisões nessa esfera de governo, permitindo a identificação das prioridades de cada município, para que com isso o gestor de saúde seja capaz de planejar os devidos procedimentos com o intuito de mitigar os danos causados pela doença a sociedade.

\title{
AGRADECIMENTOS
}

Os autores gostariam de agradecer pelo suporte financeiro (Bolsa de Iniciação Científica PIBIC/CNPq/UFPB), ao Conselho Nacional de Desenvolvimento Científico e Tecnológico (CNPq - Processo 308250/2015-0). Este trabalho também está relacionado aos Institutos Nacionais de Ciência e Tecnologia "Medicina Assistida por Computação Científica" também apoiado pelo CNPq (Processo 465586/2014-4).

\begin{abstract}
A Brasilian public health problem is dengue, whose virus is transmitted by a vector (the mosquito Aedes aegypt). Despite of efforts of health authorities in order to control this disease, the registered cases number has increased over the years. As consequence, we have an epidemiological problem of identifying priority areas to combat it providing support the definition of specific public health policies. This paper aims at the development of a spatial decision support system, which allows to take into account different information aggregated by a fuzzy rule-based system to detect priority areas for intervention on this disease. The system was applied to study of dengue at Paraíba state of Brazil from 2009 to 2013.
\end{abstract}

Keywords: Fuzzy Logic, Spatial Decision Suppor System, Dengue Fever.

\section{REFERÊNCIAS}

[1] L. Anselin. Spatial Data Analysis with GIS: an Introduction to Application in the Social Sciences. (1992).

[2] M.L. Barreto \& M.d.G.L.C. Teixeira. Dengue no Brasil: Situação Epidemiológica e Contribuições para uma Agenda de Pesquisa. (2008).

[3] J. Besag \& J. Newell. The Detection of Clusters in Rare Diseases. Journal of the Royal Statistical Society. Series A (Statistics in Society), (1991), 143-155.

[4] J.W. Coulston \& K.H. Riitters. Geographic Analysis of Forest Health Indicators Using Spatial Scan Statistics. Environmental Management, 31(6) (2003), 764-773.

[5] P.C.d.H. Furtado, I.d.S. Bezerra, I.C.A. de Souza \& R.M. de Moraes. A Estrutura Viária na Evolução do Dengue na Paraíba. In "Anais do V Congresso Brasileiro de Pesquisas Ambientais e Saúde” (2005). 
[6] G1. Brasil tem queda de 44\% nos casos de dengue; 7 estados registram alta (2012). URL Brasiltemquedade44 $\$ noscasosdedengue; 7estadosregistramalta. [Online; accessed 15-Maio-2016].

[7] Z.X. Guo, E.W.T. Ngai, C. Yang \& X. Liang. An RFID-based Intelligent Decision Support System Architecture for Production Monitoring and Scheduling in a Distributed Manufacturing Environment. International Journal of Production Economics, 159 (2015), 16-28.

[8] E.A. Henchal \& J.R. Putnak. The Dengue Viruses. Clinical Microbiology Reviews, 3(4) (1990), 376396.

[9] D.C.S.C. Holmes, R.M. Moraes \& R.P.T. Vianna. A Rule for Combination of Spatial Clustering Methods. In "The Seventh International Conferences on Pervasive Patterns and Applications" (2015).

[10] P.B. Keenan. Spatial Decision Support Systems. Decision Making Support Systems Achievements and Challenges for the New Decade, (2003), 28-39.

[11] G.C. Kelly, E. Hale, W. Donald, W. Batarii, H. Bugoro, J. Nausien, J. Smale, K. Palmer, A. Bobogare, G. Taleo et al. A High-Resolution Geospatial Surveillance-Response System for Malaria Elimination in Solomon Islands and Vanuatu. Malaria journal, 12(1) (2013), 108.

[12] M. Kulldorff \& N. Nagarwalla. Spatial Disease Clusters: Detection and Inference. Statistics in Medicine, 14(8) (1995), 799-810.

[13] B.T.L. Lucena, R.M. Moraes \& R.P.T. Vianna. Um Modelo de Suporte à Decisão Baseado em Regras Fuzzy para Predição da Segurança Alimentar. In "III Congresso Brasileiro de Sistemas Fuzzy" (2014).

[14] S.E.F. Lucena \& R.M. Moraes. Análise do Desempenho dos Métodos Scan e Besag e Newell para Identificação de Conglomerados Espaciais do Dengue no Município de João Pessoa entre os meses de Janeiro de 2004 e Dezembro de 2005. Boletim de Ciências Geodésicas, 15(4) (2009), 544-561.

[15] E.H. Mamdani \& S. Assilian. An Experiment in Linguistic Synthesis with a Fuzzy Logic Controller. International journal of Man-machine Studies, 7(1) (1975), 1-13.

[16] J.C.S. Melo, A.C.O. Melo \& R.M. Moraes. Comparação dos Métodos Scan Circular e Flexível na Detecção de Aglomerados Espaciais de Dengue. In "Escola de Informática Teórica e Métodos Formais" (2016)

[17] R.M. Moraes \& A.C.O. Melo. Sistemas de Suporte à Decisão Espacial e Aplicações. Comunicações em Informática, 1(1) (2017), 2-5.

[18] R.M. Moraes, J.A. Nogueira \& A.C.A. Souza. A New Architecture for a Spatio-Temporal Decision Support System for Epidemiological Purposes. In "11th International FLINS Conference on Decision Making and Soft Computing” (2014), pp. 17-23.

[19] S. Rozenfeld. "Fundamentos da Vigilância Sanitária". SciELO-Editora FIOCRUZ (2000).

[20] L.R. Sá. "Um Sistema de Suporte a Tomada de Decisão Espacial Usando o Perfil Demográfico para o Controle da Tuberculose no Município de João Pessoa-PB”. Master's thesis, UFPB, Universidade Federal da Paraíba, João Pessoa, PB (2015). 
[21] S. Schockaert, M. De Cock \& E.E. Kerre. "Reasoning about Fuzzy Temporal and Spatial Information from the Web", volume 3. World Scientific (2011).

[22] J.S. Silva, I. Scopel et al. A Dengue no Brasil e as Políticas de Combate ao Aedes aegypti: da Tentativa de Erradicação às Políticas de Controle. Hygeia, 4(6) (2008).

[23] I.C.A.d. Souza, R.P.d.T. Vianna \& R.M.d. Moraes. Modelagem da Incidência do Dengue na Paraíba, Brasil, por Modelos de Defasagem Distribuída. Cadernos de Saúde Pública, 23(11) (2007), 26232630.

[24] M. Sugeno \& G. Kang. Structure Identification of Fuzzy Model. Fuzzy Sets and Systems, 28(1) (1988), 15-33.

[25] R. Sugumaran, J. Degroote \& V. Sugumaran. "Spatial Decision Support Systems". Taylor \& Francis (2011).

[26] T. Tango \& K. Takahashi. A Flexibly Shaped Spatial Scan Statistic for Detecting Clusters. International Journal of Health Geographics, 4(1) (2005), 11.

[27] L.A. Zadeh. Fuzzy sets. Information and Control, 8 (1965), 338-353.

[28] L.A. Zadeh. Fuzzy logic. Computer, 21(4) (1988), 83-93. 